\title{
Ultrasound Localization of the Femoral Vein Facilitates Successful Cannulation for Hemodialysis
}

\author{
King-Kwan Lam, Hwee-Yeong Ng, Chien-Hsing Wu, Men-Tai Wu, Jin-Bor Chen, Chien-Te Lee
}

Background: Real-time ultrasound-guided techniques allow for improved cannulation of the internal jugular vein and femoral vein for hemodialysis; however, these techniques require extra sterilization procedures, specialized probes, or needle guides. A simpler ultrasound vessel localization method was performed to investigate whether this alternative approach would aid in the cannulation of the femoral vein for patients in whom temporary angioaccess was required for hemodialysis.

Methods: Patients requiring temporary femoral vein catheters for hemodialysis were divided into 2 groups on alternating days of the week during a 6-month period. One group underwent ultrasound localization of the femoral vein before cannulation and the second group received conventional landmark localization. Data regarding the strength of the

\section{At a Glance Commentary \\ Scientific background of the subject}

Real-time ultrasound-guided techniques improve cannulation of the femoral vein for hemodialysis. However, these techniques require extra sterilization procedures, specialized probes, or needle guides.

\section{What this study adds to the field}

Results of this study show that vessel localization method is a good alternative to real-time ultrasound-guided techniques and is simpler.

Results: $\quad$ Ultrasound localization resulted in significantly improved first-attempt success rates, reduced attempts, and reduced failure and complication rates overall $(p<0.001, p<0.001, p=0.002$ and $p=0.004$ respectively) as well as in the group of patients with a clearly discernible arterial pulse $(p<0.001, p=0.001$, $p=0.004$ and $p=0.011$ respectively). The same trend was observed among patients with faintly palpable or non-palpable femoral arterial pulses, although the differences were not statistically significant.

Conclusion: Cannulation of the femoral vein for hemodialysis should be performed with the aid of ultrasound. If real-time ultrasound-guided cannulation is not available, the vessel localization method is a good alternative, given its known limitations and the fact that it is simpler. It remains to be determined whether 1 - dimensional localization or localization including vessel depth information can improve outcomes in patients with faintly palpable or non-palpable femoral arterial pulses.

(Biomed J 2013;36:237-242)

Key words: cannulation, femoral vein, hemodialysis, ultrasound

$\mathrm{M}$ any conditions require hemodialysis; however, permanent angioaccess is not available in all patients. This most commonly occurs in patients with acute renal failure. Sudden loss of function of the permanent angioaccess in patients with chronic uremia is also a significant problem. Temporary angioaccess for hemodialysis usually requires cannulation of one of the central veins, namely the internal jugular vein, subclavian vein, or femoral vein. With excep- tion, ${ }^{[1]}$ the subclavian vein approach is seldom used due to the risk of subsequent venous thrombosis and stenosis. ${ }^{[2-4]}$ The internal jugular vein is usually suggested as the preferred cannulation site,${ }^{[5]}$ although cannulation at this site is technically more complicated and potentially associated with more serious complications ${ }^{[6-8]}$ than is cannulation of the femoral vein. Importantly, the notion that femoral catheterization is associated with a higher incidence of infection

From the Division of Nephrology, Department of Internal Medicine, Kaohsiung Chang Gung Memorial Hospital and Chang Gung University College of Medicine, Kaohsiung, Taiwan

Received: May 8, 2012; Accepted: Oct. 3, 2012

Correspondence to: Dr. Chien-Te Lee, Division of Nephrology, Department of Internal Medicine, Kaohsiung Chang Gung Memorial Hospital. 123, Dapi Rd., Niaosong, Kaohsiung 833, Taiwan (R.O.C.). Tel: 886-7-7317123; ext. 8306; Fax: 886-7-7322402; E-mail: chientel@gmail.com

DOI: $10.4103 / 2319-4170.113193$ 
is not supported by recent reports..$^{[9,10]}$ The femoral vein is even preferred for bedridden, coagulopathic, unstable patients ${ }^{[11]}$ and patients in whom upper-body central vein cannulation has failed. ${ }^{[12]}$

Real-time ultrasound-guided techniques improve cannulation of both the internal jugular vein ${ }^{[13-15]}$ and the femoral vein. ${ }^{[16-18]}$ However, such techniques require extra sterilization procedures, specialized probes, or needle guides, which may not be readily available in many hemodialysis units. A simpler ultrasound vessel localization technique was initially used in the internal jugular vein ${ }^{[14,19]}$ and the subclavian vein, ${ }^{[20]}$ with equivocal results. The following study was performed to investigate whether this simpler approach is beneficial for cannulation of the femoral vein in patients requiring temporary angioaccess for hemodialysis.

\section{METHODS}

Patients requiring a temporary femoral vein catheter for hemodialysis treatment in our hospital were divided into 2 groups on alternating days of the week during a 6-month period. Patients that required insertion of a temporary femoral catheter for hemodialysis and were seen on Monday, Wednesday or Friday underwent ultrasound localization of the femoral vein before the procedure. Patients seen on Tuesday, Thursday, Saturday, or Sunday underwent the conventional procedure. Written consent was obtained from all patients that underwent cannulation and hemodialysis procedures. Patients who underwent ultrasound localization of the femoral vein were informed of the procedure and agreements were obtained orally, without extra written consent. If an agreement was not obtained for the ultrasound procedure, the patient was automatically transferred to the landmark localization group.

Ultrasound localization of the femoral vein was performed in the nephrology unit's sonography room, located next to the hemodialysis unit. Patients in the ultrasound localization group received Doppler ultrasound localization of the femoral vein with a flat $10 \mathrm{MHz}$ linear array probe (Gateway, Diasonics Ultrasound Inc, U.S.A.) immediately before the cannulation procedure. The femoral vein was identified anatomically by its thin wall, easy compressibility, and the direction of blood flow. Its longitudinal course was marked as a line with ink on the skin along the probable presumed site of venous puncture. The mark was made perpendicular to the vein beneath. The patient was instructed not to move his/her marked lower extremity, especially the thigh portion, and was transferred immediately to the hemodialysis room for the cannulation procedure. To avoid variability, all ultrasound localization procedures were performed by a single operator who was not involved in the cannulation process.
After standard sterilization and local anesthesia were performed, an 18-gauge introducer needle was used to locate the femoral vein, following the ink mark guide, approximately $2 \mathrm{~cm}$ below the inguinal ligament. After confirmation of successful vein entry, a guidewire was introduced through the introducer needle. The needle was withdrawn when free to-and-fro movement of the guidewire was confirmed. Next, the skin entry site was enlarged by a scalpel before inserting a dilator. After adequate dilation, a dual lumen dialysis catheter was placed over the wire to complete the cannulation process. The procedure for the landmark localization group was identical, but was performed without the guide of the skin mark. Details of the cannulation procedure have been described previously. ${ }^{[21]}$

All of the operators that performed the cannulation procedures were nephrology fellows with comparable experience in central vein cannulation (between 3 to 5 years of experience). Junior fellows were in their fourth year of residency and had 3 to 4 years of experience in central vein cannulation. Senior fellows were in their fifth year of residency and had 4 to 5 years of experience in central vein cannulation. The cannulation procedures were performed by fellows according to a duty schedule arranged on an equality basis; and this schedule was designed independently of the study. The strength of the femoral artery pulse was assessed by the operator before cannulation and was classified as clearly discernible, faintly discernible, or non-palpable. The number of attempts was recorded as the number of skin entries and removals of the introducer needle. Failure was defined as the inability to establish a successful cannulation for hemodialysis, irrespective of the number of attempts. Complications were recorded as arterial puncture, presence of hematoma, and severe pain (requiring analgesics). Complications were evaluated for each patient during the cannulation and hemodialytic procedures and on the day after the procedures. Outcomes for patients who received femoral vein cannulation on both sides during the same hospitalization were counted as separate individual events. If a patient received more than 1 cannulation on the same side during the same hospitalization, the subsequent cannulation was excluded from the study. Patients were also excluded if they were less than 20 years of age or had coagulopathy. Failures were not included in the analyses of the number of attempts and number of complications.

\section{Statistical analyses}

The Statistical Package for the Social Sciences (SPSS) version 17 was used for the statistical analyses. The means of continuous variables were expressed as mean \pm standard deviation (SD). Outcomes were compared using the Student's $t$ test. Categorical variables were compared using the Chi-square $\left(\chi^{2}\right)$ and Fisher's exact test, where appropri- 
ate. A $p$ value of less than 0.05 was considered statistically significant.

\section{RESULTS}

In total 265 patients were included in the study [Table 1]. One hundred and three patients underwent ultrasound localization of the femoral vein before cannulation, and 162 patients underwent conventional landmark localization. Landmark localization was performed for 2 patients whose symptoms were considered too critical for ultrasound to be performed and agreements were not sought. These 2 patients had clearly discernible femoral arterial pulses and underwent successful cannulation with a total of 3 attempts. No complications were observed in these patients. The ultrasound localization and landmark localization groups were comparable in terms of age, gender, side of catheter insertion, and level of operator experience. The results indicated that the ultrasound localization group had a higher first-attempt success rate $(85.4 \%$ vs. $59.9 \%$, $p<0.001)$, fewer attempts $(1.23 \pm 0.63$ vs. $1.64 \pm 1.15$, $p<0.001)$, and lower failure (0\% vs. $8 \%, p=0.002)$ and complication ( $2.9 \%$ vs. $13.6 \%, p=0.004)$ rates than did the landmark localization group.
Results were further analyzed after dividing the patients into 2 groups according to whether the femoral arterial pulse was readily discernible. In the group with clearly discernible femoral arterial pulses [Table 2], the ultrasound localization group showed a higher first-attempt success rate $(88.5 \%$ vs. $63.3 \%, p<0.001)$, fewer attempts $(1.20 \pm 0.61$ vs. $1.59 \pm 1.17, p=0.001)$, and lower failure (0\% vs. $8.2 \%$, $p=0.004)$ and complication $(3.1 \%$ vs. $12.9 \%, p=0.011)$ rates than those of the landmark localization group. The same trend of results existed for the faintly discernible and non-palpable femoral arterial pulse group [Table 3], although the differences were not statistically significant.

Table 4 shows the distribution of complication events among the patients. In patients with clearly discernible femoral arterial pulses, 5 events ( 3 arterial punctures and 2 hematomas) occurred in 3 patients who underwent the ultrasound localization procedure. In total 22 events ( 11 arterial punctures, 9 hematomas, and 2 episodes of severe pain) occurred in 19 patients who underwent the landmark localization procedure. For patients with faintly discernible or non-palpable femoral arterial pulses, no complications were observed in those who underwent ultrasound localization. Five events (1 arterial puncture, 2 hematomas, and 2 episodes of severe pain) occurred

Table 1: Overall comparison between the ultrasound localization and landmark localization groups as seen in the study

\begin{tabular}{|c|c|c|c|c|c|c|c|c|c|}
\hline & $\begin{array}{l}\text { Number } \\
(n=265)\end{array}$ & Age & $\begin{array}{l}\text { Sex: } \\
\text { Male }\end{array}$ & $\begin{array}{l}\text { Right } \\
\text { side }\end{array}$ & $\begin{array}{c}\text { Operator } \\
\text { experience: Junior } \\
\text { fellow }\end{array}$ & $\begin{array}{c}\text { First } \\
\text { attempt } \\
\text { successful }\end{array}$ & $\begin{array}{l}\text { Number of } \\
\text { attempts } \\
(n=252)\end{array}$ & $\begin{array}{l}\text { Number of } \\
\text { failures }\end{array}$ & $\begin{array}{c}\text { Number of } \\
\text { complications } \\
(n=252)\end{array}$ \\
\hline$\overline{\mathrm{UL}}$ & 103 & $58.48 \pm 13.83$ & $48(46.6 \%)$ & $81(78.6 \%)$ & $45(43.7 \%)$ & $88(85.4 \%)$ & $1.23 \pm 0.63$ & $0(0 \%)$ & $3(2.9 \%)$ \\
\hline LM & 162 & $57.92 \pm 14.91$ & $64(39.5 \%)$ & $112(69.1 \%)$ & $80(49.4 \%)$ & $97(59.9 \%)$ & $1.64 \pm 1.15$ & $13(8.0 \%)$ & $22(13.6 \%)$ \\
\hline$p$ value & & 0.761 & 0.308 & 0.119 & 0.380 & $<0.001$ & $<0.001$ & 0.002 & 0.004 \\
\hline
\end{tabular}

Abbreviations: UL: Ultrasound localization; LM: Landmark localization

Table 2: Comparison of results between the ultrasound localization and landmark localization groups in patients with clearly discernible femoral arterial pulse as seen in the study

\begin{tabular}{lccccccccc}
\hline & $\begin{array}{c}\text { Number } \\
(n=243)\end{array}$ & Age & $\begin{array}{c}\text { Sex: } \\
\text { Male }\end{array}$ & $\begin{array}{c}\text { Right } \\
\text { side }\end{array}$ & $\begin{array}{c}\text { Operator } \\
\text { experience: Junior } \\
\text { fellow }\end{array}$ & $\begin{array}{c}\text { First } \\
\text { attempt } \\
\text { successful }\end{array}$ & $\begin{array}{c}\text { Number of } \\
\text { attempts } \\
(n=231)\end{array}$ & $\begin{array}{c}\text { Number of } \\
\text { failures }\end{array}$ & $\begin{array}{c}\text { Number of } \\
\text { complications } \\
(n=231)\end{array}$ \\
\hline UL & 96 & $58.10 \pm 14.08$ & $46(47.9 \%)$ & $76(79.2 \%)$ & $42(43.8 \%)$ & $85(88.5 \%)$ & $1.20 \pm 0.61$ & $0(0 \%)$ & $3(3.1 \%)$ \\
LM & 147 & $57.99 \pm 14.09$ & $59(40.1 \%)$ & $103(70.1 \%)$ & $72(49.0 \%)$ & $93(63.3 \%)$ & $1.59 \pm 1.17$ & $12(8.2 \%)$ & $19(12.9 \%)$ \\
$p$ value & & 0.951 & 0.237 & 0.137 & 0.434 & $<0.001$ & 0.001 & 0.004 & 0.011 \\
\hline
\end{tabular}

Abbreviations: UL: Ultrasound localization; LM: Landmark localization

Table 3: Comparison of results between the ultrasound localization and landmark localization groups in patients with faintly or non-palpable femoral arterial pulse as seen in the study

\begin{tabular}{lccccccccc}
\hline & $\begin{array}{c}\text { Number } \\
(n=22)\end{array}$ & Age & $\begin{array}{c}\text { Sex: } \\
\text { Male }\end{array}$ & $\begin{array}{c}\text { Right } \\
\text { side }\end{array}$ & $\begin{array}{c}\text { Operator } \\
\text { experience: Junior } \\
\text { fellow }\end{array}$ & $\begin{array}{c}\text { First } \\
\text { attempt } \\
\text { successful }\end{array}$ & $\begin{array}{c}\text { Number of } \\
\text { attempts } \\
(n=21)\end{array}$ & $\begin{array}{c}\text { Number of } \\
\text { failures }\end{array}$ & $\begin{array}{c}\text { Number of } \\
\text { complications } \\
(n=21)\end{array}$ \\
\hline $\mathrm{UL}$ & 7 & $63.57 \pm 9.16$ & $2(28.6 \%)$ & $5(71.4 \%)$ & $3(42.9 \%)$ & $3(42.9 \%)$ & $1.71 \pm 0.76$ & $0(0 \%)$ & $0(0 \%)$ \\
LM & 15 & $57.27 \pm 13.53$ & $5(33.3 \%)$ & $9(60.0 \%)$ & $8(53.3 \%)$ & $4(26.7 \%)$ & $2.14 \pm 0.86$ & $1(6.7 \%)$ & $3(20 \%)$ \\
$p$ value & & 0.279 & 1.000 & 0.137 & 0.460 & 0.630 & 0.280 & 1.000 & 0.523 \\
\hline
\end{tabular}

Abbreviations: UL: Ultrasound localization; LM: Landmark localization 
Table 4: Distribution of complication events in patients using the ultrasound localization and landmark localization methods classified by whether the femoral arterial pulse was readily palpable as seen in the study

\begin{tabular}{|c|c|c|c|c|c|c|c|}
\hline & \multirow[t]{2}{*}{ Number of patients } & \multicolumn{3}{|c|}{ Clearly discernible femoral arterial pulse* } & \multicolumn{3}{|c|}{ Faintly or non-palpable femoral arterial pulse ${ }^{\dagger}$} \\
\hline & & Arterial puncture & Hematoma & Pain & Arterial puncture & Hematoma & Pain \\
\hline$\overline{\mathrm{UL}}$ & 3 & 3 & 2 & 0 & 0 & 0 & 0 \\
\hline LM & 22 & 11 & 9 & 2 & 1 & 2 & 2 \\
\hline
\end{tabular}

Abbreviations: UL: Ultrasound localization; LM: Landmark localization; *A total of 5 events of complications occurred in 3 patients (2 patients had 2 complications) in the UL group; 22 events of complications occurred in 19 patients ( 3 patients had 2 complications) in the LM group; ${ }^{\dagger} \mathrm{No}$ complication occurred in the UL group; 5 events of complications occurred in 3 patients (1 patient had 3 complications) in the LM group

in 3 patients who underwent the conventional landmark localization procedure.

\section{DISCUSSION}

In clinical medicine, central venous cannulation is a common practice for uremic patients requiring hemodialysis treatment without permanent angioaccess. Although the procedures are generally considered easy, they are not without risks. The complication rates of previous reports were $7.7 \%$ to $11.6 \%{ }^{[13,17,22]}$ for the internal jugular vein and $1 \%$ to $12.2 \%^{[1,23,24]}$ for the subclavian vein, depending on the definitions of complications and experience levels of operators. Of note, a high incidence of subsequent venous thrombosis and stenosis were reported for the subclavian vein approach. Failure rates were reported to be $10 \%$ to $18 \%^{[13,17,22]}$ for the internal jugular vein and $3 \%$ to $16.5 \%^{[1,23]}$ for the subclavian vein. Although the success rates were better with the femoral vein approach $(80 \%$ to $100 \%){ }^{[16,18]}$ the complication rates were between $9 \%$ to $18.4 \%,{ }^{[11,16,18]}$ depending on the definitions of complications and operator experience.

Real-time ultrasound-guided cannulation of the femoral vein improved the success rate and reduced the time to access, number of needle passes, and complications. Favorable results were reported for both dialytic ${ }^{[11,16,18]}$ and non-dialytic ${ }^{[25]}$ procedures, and some centers routinely perform this procedure. ${ }^{[21]}$

Although real-time ultrasound-guided cannulation of the femoral vein can be performed by a single experienced operator, some centers found it necessary to perform the procedure with the cooperation of 2 persons. ${ }^{[14,25]}$ To maintain aseptic conditions at the operation site, a special sterile sheath is wrapped around the ultrasound probe. Specialized probes or needle guides are used in some centers. These may pose special problems and add complexities to the procedure. The skin mark method used in our study is simpler and can be performed in all units that have an ultrasound with a suitable frequency probe. After marking the position of the femoral vein, the remainder of the procedure is identical to the traditional landmark method. Importantly, the ultrasound localization group had higher first-attempt success rates, fewer attempts, higher overall success rates, and fewer complications than those of the landmark localization group.

Although the venous localization method is not new, its use has not been reported for femoral vein cannulation. The method has been used for cannulation of the internal jugular vein ${ }^{[14,19]}$ and subclavian vein, ${ }^{[20]}$ with equivocal benefits. Legler et al., found that Doppler localization of the internal jugular vein improved the single-pass success rate, ${ }^{[19]}$ although the sample size was small and the complication rate was not reported. Mansfield et al., performed a well-designed study and commented that ultrasound guidance had no effect on the rate of complications or failures in subclavian vein catheterization. ${ }^{[20]}$ Nevertheless, as the authors noted, they had a large number of operators with variable experience, and catheterizations were performed mostly for cancer patients with histories of operations or radiotherapy. The level of experience of the operator in central vein cannulation is important, ${ }^{[18,22,26]}$ therefore the conclusions of Mansfield et al., should be viewed conservatively when considering patients without cancer. Nadig et al., compared the skin mark method with real-time ultrasound-guided cannulation of the internal jugular vein using the stringent criteria of the number of attempts as a function of the forward movement of both the local anesthesia needle ( 22 gauge) and the introducer needle (18 gauge), instead of the number of entries of the introducer needle, as is used in many studies. ${ }^{[14]}$ In their study, real-time ultrasound guidance resulted in significantly fewer unsuccessful attempts without adding additional time. Differences were induced primarily by movement of the patient's head before cannulation after making the skin mark. For most patients, it is likely much more difficult to keep the head fixed in a lateral position during the entire procedure than the thigh. Although we did not directly compare our method with the real-time ultrasound-guided method, our results were comparable to those of a recently published report by Prabhu et al., that used real-time ultrasound guidance (overall success rates, $100 \%$ vs. $98.2 \%$; first-attempt success rates, $85.4 \%$ vs. $85.5 \%$; number of attempts, $1.23 \pm 0.63$ vs. $1.16 \pm 0.42$; and complication rates, $2.9 \%$ vs. $5.5 \%$ in the ultrasound localization group and real-time ultrasound-guided group, respectively). ${ }^{[18]}$

Variations in the location of femoral veins are relatively rare, ${ }^{[27]}$ whereas changes in the relative positions of the femoral artery and vein were noted with different positions of the 
leg. ${ }^{[28,29]}$ From the clinical viewpoint, it was also suggested that even in ultrasound-guided cases, abduction of the leg might enhance vein entry. ${ }^{[21]}$ This may explain why patients with clearly discernible femoral arterial pulses benefited from ultrasound localization of the femoral vein. Seyahi et al., concluded that ultrasound-guided cannulation should be the preferred method for patients with non-palpable femoral artery pulses. ${ }^{[27]}$ Overweight and particularly obese patients are also candidates. Our data from the faintly discernible or non-palpable femoral arterial pulse group was compatible with such observations, although differences between the ultrasound localization and the landmark localization groups were not statistically significant. One reason for this difference might be that the number of patients in the faintly discernible and non-palpable femoral arterial pulse groups was small. Another explanation may be that this group of patients was mostly obese and edematous. Of note, ultrasound localization of the femoral vein in this study was only 1-dimensional, and the depth of the femoral vessels was not measured. This may be very important factor to consider, as vessels of obese patients lie deeper than those of non-obese patients. ${ }^{[27]} \mathrm{A}$ modified method, including measurement of the depth of the vessels, might be required for obese and edematous patients.

To evaluate complications, patients were examined during the cannulation and hemodialysis procedures. $\mathrm{Pa}-$ tients were also reexamined the day after the procedures to detect delayed onset complications. Arterial puncture and hematoma occurred early after the cannulations. Five patients (only 4 patients shown in Table 4 because 1 patient in the clearly discernible femoral arterial pulse group was excluded due to failure of cannulation) complained of severe pain at the cannulation site after cessation of the effects of local anesthesia. Such pain might be caused by inadvertent punctures, deep-seated hematoma, or abnormal angulation of the dialysis catheter, all of which may have been overlooked by previous observers. We suggest monitoring for these complications, especially after difficult cannulations.

Despite the favorable results, the implications of our findings should be considered in light of the following caveats. Although success and complication rates were assessed, the time spent to establish a successful cannulation were not assessed and compared between each method. We found that the technique of vessel localization was easy to learn and perform; however, on 2 occasions, direct cannulation using the landmark method was more appropriate in emergency settings. We did not attempt to fix the marked lower extremity after venous localization other than to transfer the patient immediately for the cannulation process. The beneficial effects observed might be evidence that significant deviation of the skin mark did not occur. However, one should be conservative if there is a significant delay between making the skin mark and performing the cannulation procedure, especially when movement of the marked lower extremity has occurred. A bedside portable ultrasound system may help reduce movement before the cannulation procedure.

Our results, taken together with the results of previous reports regarding real-time ultrasound-guided cannulation, suggest that cannulation of the femoral vein for hemodialysis should be performed with the aid of ultrasound. This method improves the overall success rates and first-attempt success rates while reducing the number of attempts and complications. If real-time ultrasound-guided cannulation is not available, vessel localization is a good alternative, given that its limitations are known and it is simpler. The utility of this method for patients with faintly palpable or non-palpable femoral arterial pulses remains to be demonstrated.

\section{REFERENCES}

1. Karkee DV. Subclavian vein dialysis access catheter-complications are low. Nepal Med Coll J 2010;12:248-52.

2. Davis D, Petersen J, Feldman R, Cho C, Stevick CA. Subclavian venous stenosis. A complication of subclavian dialysis. JAMA 1984;252:3404-6.

3. Vanherweghem JL, Yassine T, Goldman M, Vandenbosch G, Delcour C, Struyven J, et al. Subclavian vein thrombosis: A frequent complication of subclavian vein cannulation for hemodialysis. Clin Nephrol 1986;26:235-8.

4. Clark DD, Albina JE, Chazan JA. Subclavian vein stenosis and thrombosis: A potential serious complication in chronic hemodialysis patients. Am J Kidney Dis 1990;15:265-8.

5. Schetz M. Vascular access for HD and CRRT. Contrib Nephrol 2007;156:275-86.

6. Ahmad M, Hayat A. Horner's syndrome following internal jugular vein dialysis catheter insertion. Saudi J Kidney Dis Transpl 2008;19:94-6.

7. Li H, Wang SX, Wang W, Xu C, Shen S, Yu L, et al. Thrombosis of the superior vena cava and auxiliary branches in patients with indwelling catheterization of the internal jugular vein. Chin Med J-Peking 2009;122:692-6.

8. Lee YM, Kim HJ, Lee JE, Song JH, Lee MK, Lee SY, et al. Cardiac tamponade following insertion of an internal jugular vein catheter for hemodialysis. Clin Nephrol 2009;72:220-3.

9. Stolic R, Trajkovic G, Peric V, Jovanovic A, Stolic D, Sovtic S, et al. Central venous catheters in hemodialysis: To accept recommendations or to stick to own experience. Vojnosanit Pregl 2008;65:21-6.

10. Casanegra AI, Brannan S, Dadu R, Ong P, Berner M, Spinning K, et al. Short-term femoral vein catheterization rarely causes thrombosis or bacteremia. J Hosp Med (Online) 2011;6:33-6.

11. Hartle E, Conlon P, Carstens R, Schwab S. Ultrasound guided cannulation of the femoral vein for acute hemodialysis access. J Am Soc Nephrol 1993;4:352.

12. Requarth JA. Real-time ultrasound-aided central vein cannulation failure rate: Establishing a benchmark. World J Surg 2010;34:3054-8. 
13. Lin BS, Huang TP, Tang GJ, Tarng DC, Kong CW. Ultrasound-guided cannulation of the internal jugular vein for dialysis vascular access in uremic patients. Nephron 1998;78:423-8.

14. Nadig C, Leidig M, Schmiedeke T, Hoffken B. The use of ultrasound for the placement of dialysis catheters. Nephrol Dial Transpl 1998;13:978-81.

15. Bansal R, Agarwal SK, Tiwari SC, Dash SC. A prospective randomized study to compare ultrasound-guided with nonultrasound-guided double lumen internal jugular catheter insertion as a temporary hemodialysis access. Renal Fail 2005;27:561-4.

16. Kwon TH, Kim YL, Cho DK. Ultrasound-guided cannulation of the femoral vein for acute haemodialysis access. Nephrol Dial Transpl 1997; 12:1009-12.

17. Farrell J, Gellens M. Ultrasound-guided cannulation versus the landmark-guided technique for acute haemodialysis access. Nephrol Dial Transpl 1997;12:1234-7.

18. Prabhu MV, Juneja D, Gopal PB, Sathyanarayanan M, Subhramanyam S, Gandhe S, et al. Ultrasound-guided femoral dialysis access placement: A single-center randomized trial. Clin J Am Soc Nephrol 2010;5:235-9.

19. Legler D, Nugent M. Doppler localization of the internal jugular vein facilitates central venous cannulation. Anesthesiology 1984;60:481-2.

20. Mansfield PF, Hohn DC, Fornage BD, Gregurich MA, Ota DM. Complications and failures of subclavian-vein catheterization. N Engl J Med 1994;331:1735-8.

21. Pervez A, Abreo K. Techniques and tips for quick and safe temporary catheter placement. Semin Dial 2007;20:621-5.

22. Denys BG, Uretsky BF, Reddy PS. Ultrasound-assisted cannulation of the internal jugular vein. A prospective comparison to the external landmark-guided technique. Circulation 1993;87:1557-62.

23. Vanherweghem JL, Cabolet P, Dhaene M, Goldman M, Stolear JC, Sabot JP, et al. Complications related to subclavian catheters for hemodialysis. Report and review. Am J Nephrol 1986;6:339-45.

24. Kamran T, Zaheer K, Khan AA, Khalid M, Akhtar MS. Applications and complications of subclavian vein catheterization for hemodialysis. J Coll Physicians Surg Pak 2003;13:40-3.

25. Iwashima $\mathrm{S}$, Ishikawa $\mathrm{T}$, Ohzeki $\mathrm{T}$. Ultrasound-guided versus landmark-guided femoral vein access in pediatric cardiac catheterization. Pediatr Cardiol 2008;29:339-42.

26. Lockwood AH. Percutaneous subclavian vein catheterization. Too much of a good thing? Arch Intern Med 1984;144:1407-8.

27. Seyahi N, Kahveci A, Altiparmak MR, Serdengecti K, Erek E Ultrasound imaging findings of femoral veins in patients with renal failure and its impact on vascular access. Nephrol Dial Transpl 2005;20:1864-7.

28. Hopkins JW, Warkentine F, Gracely E, Kim IK. The anatomic relationship between the common femoral artery and common femoral vein in frog leg position versus straight leg position in pediatric patients. Acad Emerg Med 2009;16:579-84.

29. Werner SL, Jones RA, Emerman CL. Effect of hip abduction and external rotation on femoral vein exposure for possible cannulation. J Emerg Med 2008;35:73-5. 